\title{
Observation of energetic electrons within magnetic islands
}

\author{
L.-J. CHEN ${ }^{1 *}$, A. BHATTACHARJEE ${ }^{1}$, P. A. PUHL-QUINN1 ${ }^{1}$, H. YANG ${ }^{1}$, N. BESSHO ${ }^{1}$, S. IMADA², \\ S. MÜHLBACHLER ${ }^{3}$, P. W. DALY ${ }^{3}$, B. LEFEBVRE ${ }^{4}$, Y. KHOTYAINTSEV ${ }^{5}$, A. VAIVADS ${ }^{5}$, A. FAZAKERLEY6 \\ AND E. GEORGESCU7
}

\author{
${ }^{1}$ Space Science Center, University of New Hampshire, Durham, New Hampshire 03824, USA \\ ${ }^{2}$ National Astronomical Observatory of Japan, 2-21-1 Osawa, Tokyo 181-8588, Japan \\ ${ }^{3}$ Max-Planck-Institute for Solar System Research, D-37191 Katlenburg-Lindau, Germany \\ ${ }^{4}$ The Blackett Laboratory, Imperial College London, London SW7 2BW, UK \\ ${ }^{5}$ Swedish Institute of Space Physics, SE-75121 Uppsala, Sweden \\ ${ }^{6}$ Mullard Space Science Laboratory, University College London, Holmbury St Mary, Dorking RH5 6NT, UK \\ ${ }^{7}$ Max-Planck-Institute for Extraterrestrial Physics, D-85741 Garching, Germany \\ *e-mail: lijen@mailaps.org
}

Magnetic reconnection is the underlying process that releases impulsively an enormous amount of magnetic energy ${ }^{1}$ in solar flares $^{2,3}$, flares on strongly magnetized neutron stars $^{4}$ and substorms in the Earth's magnetosphere ${ }^{5}$. Studies of energy release during solar flares, in particular, indicate that up to $50 \%$ of the released energy is carried by accelerated $20-100 \mathrm{keV}$ suprathermal electrons ${ }^{6-8}$. How so many electrons can gain so much energy during reconnection has been a long-standing question. A recent theoretical study suggests that volumefilling contracting magnetic islands formed during reconnection can produce a large number of energetic electrons ${ }^{9}$. Here we report the first evidence of the link between energetic electrons and magnetic islands during reconnection in the Earth's magnetosphere. The results indicate that energetic electron fluxes peak at sites of compressed density within islands, which imposes a new constraint on theories of electron acceleration.

In situ observations have demonstrated production of energetic electrons by magnetic reconnection ${ }^{10}$. However, how electrons can be accelerated in large numbers to suprathermal energies during magnetic reconnection remains an outstanding puzzle. In the context of reconnection in the Earth's magnetotail, even the largest possible electron outflow velocity (the upstream electron Alfvén velocity $V_{\mathrm{Ae}}=B / \sqrt{4 \pi m_{\mathrm{e}} n}$, where $B$ is the magnetic field strength, $m_{\mathrm{e}}$ the electron mass and $n$ the plasma density) corresponds to electron energies of only about $2 \mathrm{keV}$. Thus, outflows driven by reconnection can in no way account for the observed suprathermal energies (of the order of 10-100 keV) of electrons.

State-of-the-art particle-in-cell (PIC) simulations of reconnection, aiming to address suprathermal electron production, show that electrons are primarily accelerated at the $\mathrm{X}$ line or separatrices (see Fig. 1) by electric fields ${ }^{11-13}$. The consequence of primary electron acceleration at the $\mathrm{X}$ line and separatrices is the emergence of energetic electrons at these sites. The PIC simulations, although fully self-consistent, are two dimensional. It is difficult to see how the mechanism of electron acceleration identified by these simulations can account for the large number of

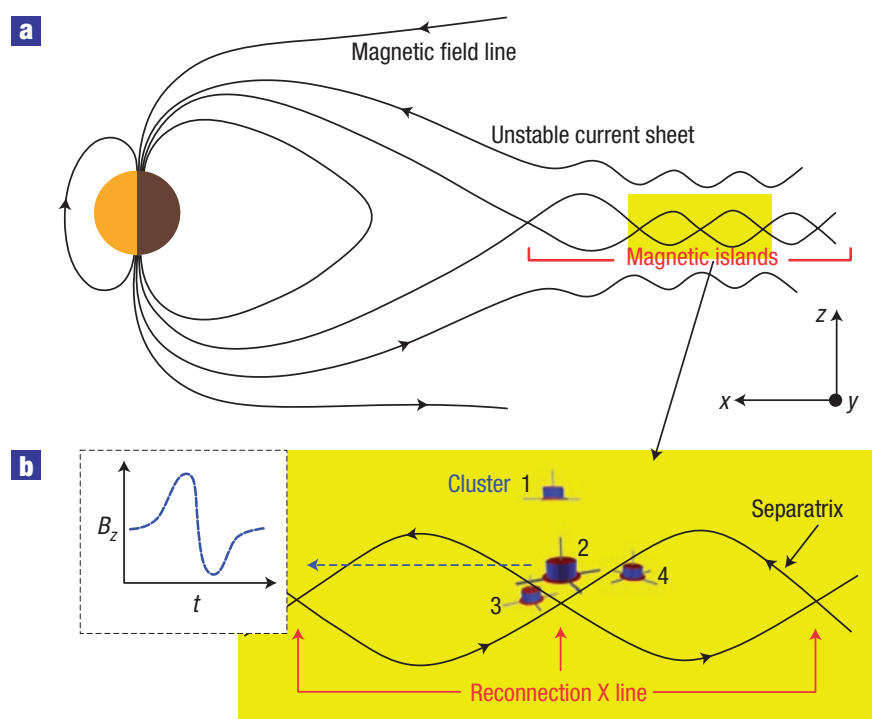

Figure 1 Schematic diagrams of magnetic islands in the Earth's magnetotail. a, The noon-midnight plane of the near-Earth magnetosphere showing multiple magnetic islands formed in a two-dimensional cartoon of the unstable magnetotail current sheet during reconnection. The coordinate system is defined such that $x$ is towards the Sun, $z$ is normal to the current sheet, and $y$ is directed out of the $x-z$ plane. $\mathbf{b}$, Signatures of magnetic islands in the $z$ component of the magnetic field $\left(B_{z}\right)$ as observed by a spacecraft. The current of the current sheet flows in the positive $y$ direction, and so do the filamentary currents within magnetic islands. The positions of the four Cluster spacecraft are sketched to depict the tetrahedron configuration (the different sizes of the spacecraft represent their displacements in $y$ as shown in Fig. $3 \mathrm{~m}$ ). The trajectory of the tetrahedron barycentre is represented by a dashed line to illustrate the relative motion of the spacecraft and island (the island moves away from the Earth). As a result of this motion, $B_{z}$ shows a bipolar signature. 


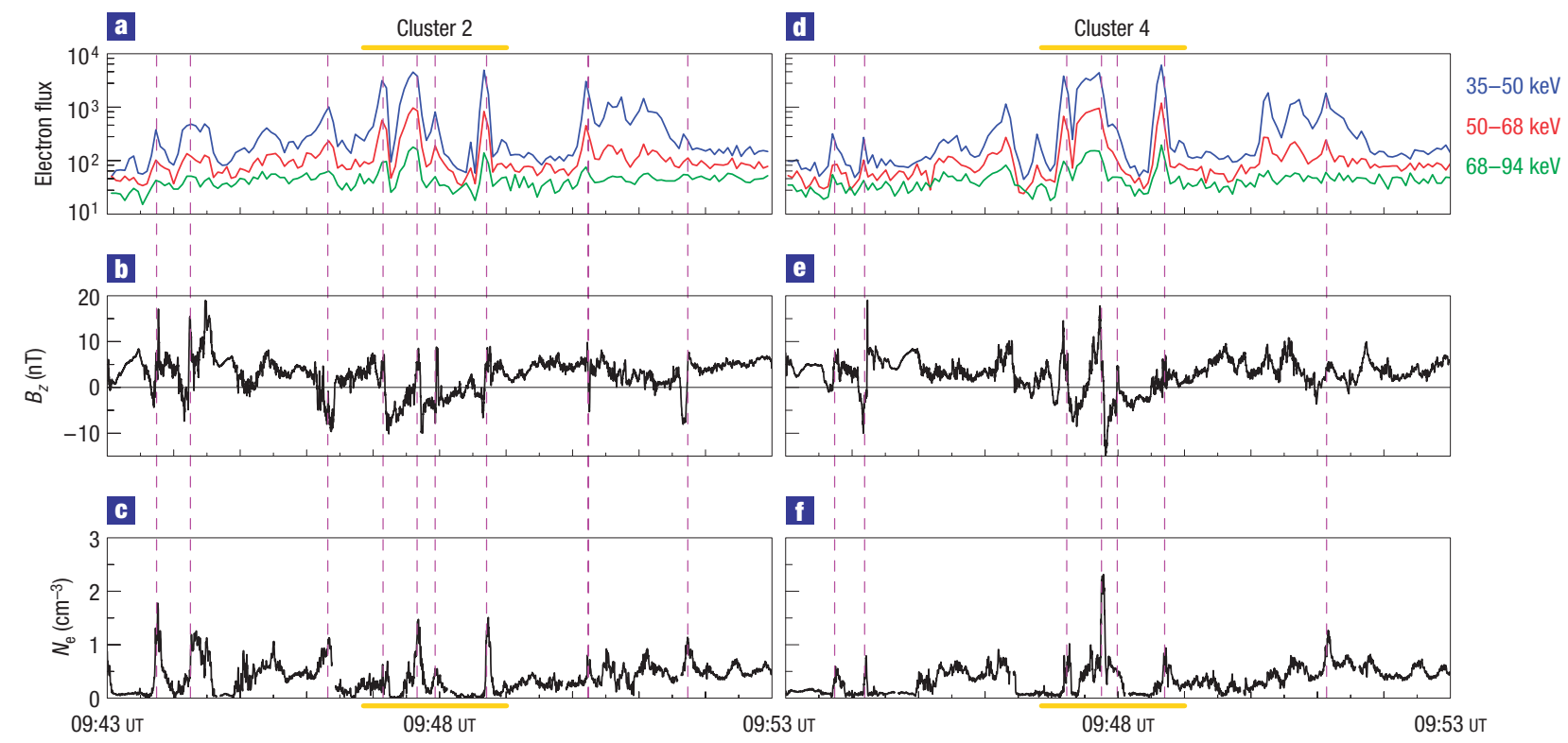

Figure 2 Association between energetic electron bursts and the signatures of magnetic islands. a,d, Differential energy fluxes (in units of $\mathrm{cm}^{-2} \mathrm{keV}^{-1} \mathrm{~s}^{-1}$ at $4 \mathrm{~s}$ resolution) of electrons with energies of 35-94 keV in three energy channels recorded by Cluster. Energetic electron bursts are seen in all three energy channels. b,e, The $z$ components of the magnetic fields $\left(B_{z}\right.$, in GSM) measured with a sampling rate of 24 vectors s ${ }^{-1}$. c,f, Electron densities $N_{\mathrm{e}}\left(5\right.$ samples s $\left.^{-1}\right)$ deduced from measurements by the double-probe electric-field-wave experiment ${ }^{25}$ on board Cluster. Localized bipolar $B_{z}$ pulses are due to relative motion of the spacecraft and magnetic islands (as illustrated by schematic diagram $\mathbf{b}$ in Fig. 1). Bipolar $B_{z}$ pulses together with enhanced electron densities are identified with magenta dashes to show their association with electron bursts.

accelerated electrons in solar flares, because the volume over which reconnection electric fields are operative is only a small fraction of the total flare volume. A recent theoretical study suggests that Fermi acceleration from volume-filling contracting magnetic islands is the dominant mechanism for acceleration in three dimensions ${ }^{9}$. The immediate observables for the operation of this latter mechanism are the existence of many magnetic islands in three dimensions, and electrons that are accelerated within the islands. Although energetic electron fluxes have been reported to peak at the crossing of a magnetotail reconnection $\mathrm{X}$ line $\mathrm{e}^{10}$, until now there has been no evidence showing any association between magnetic islands and energetic electrons. In this letter, we report the observation of a series of coherent magnetic structures, which show signatures of magnetic islands when viewed along the one-dimensional spacecraft trajectories. Each of these magnetic structures (hereafter referred to as magnetic islands) has its corresponding energetic electron burst, according to data from the four Cluster spacecraft ${ }^{14}$ during a near-Earth reconnection event. The high-time-resolution data reveal that energetic electron fluxes peak at the sites of density compression within islands, a new feature that is so far not explained by existing theories.

On 1 October 2001, multiple substorms occurred during a strong geomagnetic storm. The four Cluster spacecraft were in the magnetotail plasma sheet (see Fig. 1 for an illustration) about $17 R_{\mathrm{E}}$ from the Earth when the largest substorm commenced at around 09:30 U T. Cluster observed increased total pressure (plasma and magnetic pressure) and ion flow during the growth phase of the substorm, followed by a decrease of the pressure and cessation of the flow at onset ${ }^{15}$. About $13 \mathrm{~min}$ after the substorm onset (during the expansion phase of the substorm), Cluster started to observe bursts of energetic electrons (Fig. 2a,d). The energetic electron bursts were observed primarily by Cluster 2-4 (only data from Cluster 2 and 4 are shown), in the energy range $35-94 \mathrm{keV}$. The bursts went on and off for about $10 \mathrm{~min}$, in the midst of several flow reversals ${ }^{15}$, consistent with the passages of multiple reconnection sites. The flux profiles observed by Cluster 2 and 4 are qualitatively similar, indicating that most of the electron bursts have a spatial extent larger than the spacecraft separation (depicted in Fig. 1b), which is $\sim 1,500 \mathrm{~km}$ in $x, \sim 1,200 \mathrm{~km}$ in $y$, and $\sim 500 \mathrm{~km}$ in $z$ in geocentric solar magnetospheric (GSM) coordinates.

The magnetotail current sheet consists primarily of currents flowing from dawn to dusk. The half-width of the current sheet during the period plotted in Fig. 2 is estimated to be $\sim 1,000 \mathrm{~km}$ on the basis of the observed magnetic-field data ${ }^{15}$. This width is roughly one hydrogen ion inertial length $\left(c / \omega_{\mathrm{pi}}\right.$, where $c$ is the speed of light and $\omega_{\mathrm{pi}}$ the proton plasma frequency). A twodimensional current sheet with the above properties is potentially unstable to collisionless tearing instabilities and the formation of magnetic islands ${ }^{16}$ (filamentary structures of currents as illustrated in Fig. 1). Spacecraft passing through such magnetic islands will observe bipolar pulses in the $z$ component of the magnetic field (Fig. 1b). In addition, if reconnection continues to occur at the two $\mathrm{X}$ lines that demarcate a magnetic island, the outflow jets will pile up plasma within the island, causing the plasma density to be enhanced (Fig. 2c,f). Most of the energetic electron bursts observed by Cluster have corresponding bipolar $B_{z}$ pulses and electron density enhancements (corresponding bursts and islands are identified as magenta dashed lines in Fig. 2; see the Methods section for island identification criteria), indicating that the presence of energetic electrons is linked to time-dependent magnetic islands. During this $10 \mathrm{~min}$ interval, about 10 magnetic islands and their corresponding electron bursts were observed, evidence of electron acceleration at multiple sites.

Measurements from all four spacecraft during the period between 09:46:40 and 09:49:00 U T (highlighted with gold bars in Fig. 2 and plotted in Fig. 3), which contains the three strongest 

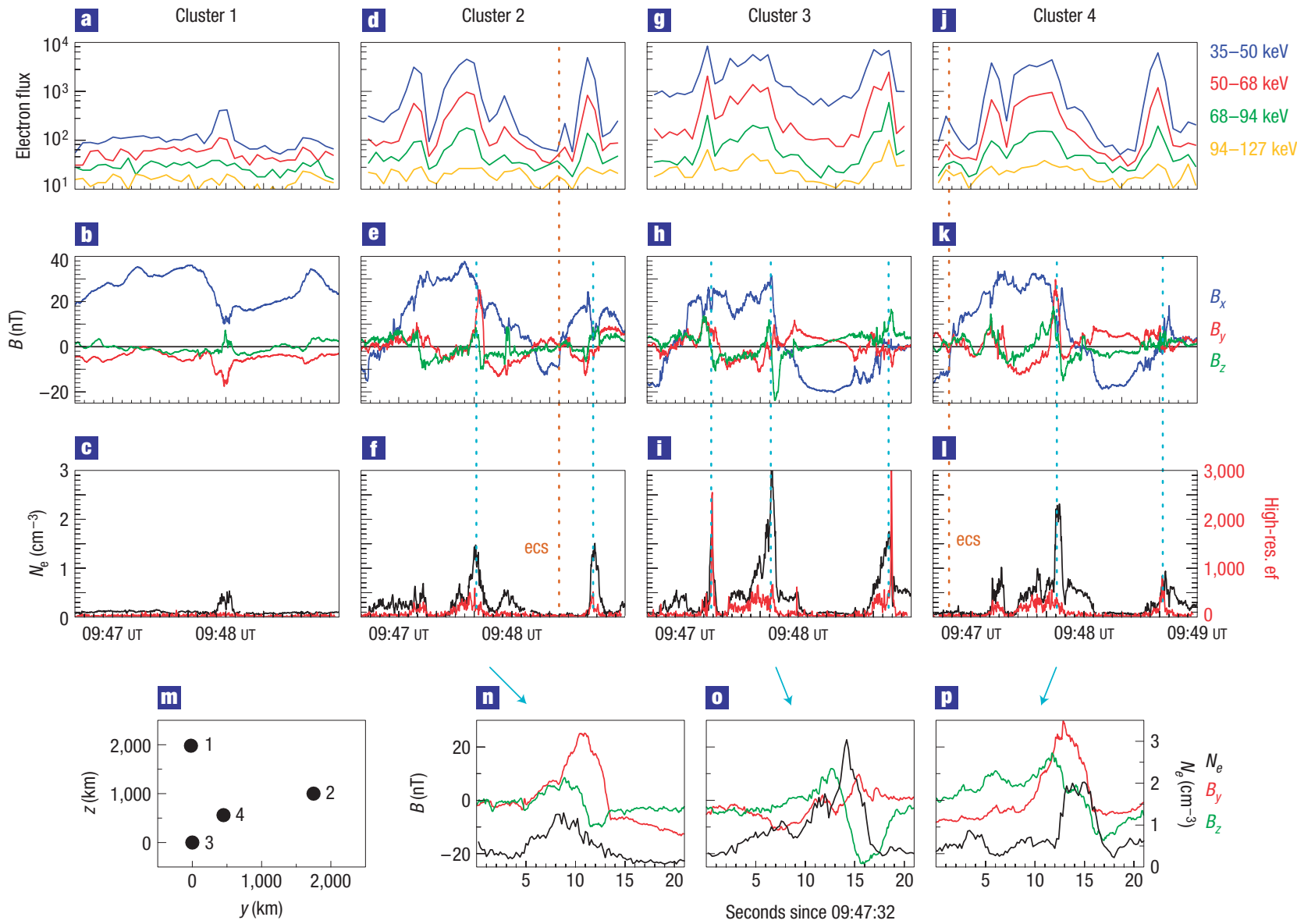

Figure 3 Four-spacecraft views of the three largest energetic electron bursts and their corresponding magnetic islands. a,d,g,j, Differential energy fluxes of electrons observed by Cluster 1-4, respectively. Two secondary peaks correspond to energetic electrons observed at the electron-current sheets (marked by dotted brown lines, and labelled as 'ecs') where the reconnection X lines reside. $\mathbf{b}, \mathbf{e}, \mathbf{h}, \mathbf{k}$, Three components of magnetic fields showing generic signatures of magnetic islands: bipolar $B_{z}$; unipolar or double-peaked $B_{y}$. c,f,i,l, Electron densities (black curves) and high-time-resolution electron fluxes for energies greater than 50 keV (ref. 26) (red curves, 4 sample s ${ }^{-1}$ ). Energetic electron fluxes peak at the density compression sites within islands (dotted cyan lines). $\mathbf{m}$, The projection of the relative spacecraft positions onto the $y-z$ (GSM) plane. $\mathbf{n , 0 , p}$, A detailed view of $B_{z}, B_{y}$ and the compressed density within a three-dimensional island observed by Cluster $2-4$. The $B_{y}$ structure observed by Cluster 3 (double peaked) differs from that observed by Cluster 2 and 4 (single peaked), implying that the in-plane current of the island varies significantly over a few hundred kilometres (a fraction of one ion inertial length) in the out-of-plane direction.

electron bursts, further substantiate the link between energetic electrons and magnetic islands. Energetic electron fluxes from all four spacecraft (Fig. 3a,d,g,j) show that the three strongest bursts were observed only by Cluster $2-4$. The flux enhancements were observed up to the energy of $127 \mathrm{keV}$. Cluster 1, being the northernmost spacecraft of the four, detected only a small electron burst (Fig. 3a) as it crossed the edge of a small island (Fig. 3b). Three components of the magnetic field recorded during the highlighted period (Fig. 3b,e,h,k) show decreases in $B_{x}$, bipolar $B_{z}$ and single-peaked and double-peaked $B_{y}$ (signatures of magnetic islands which will be discussed in the next paragraph). The out-of-plane magnetic field $B_{y}$ observed by Cluster 2 and 4 at around 09:47:40 UT (the red unipolar pulses marked by dotted cyan lines in Fig. $3 \mathrm{e}$ and $\mathrm{k}$ ) are highly correlated. This high correlation enables us to estimate the velocity of the island to be $\sim 500 \mathrm{~km} \mathrm{~s}^{-1}$ away from the Earth, and the size of the island $\sim 2,500 \mathrm{~km}$ (see the Methods section), about two ion inertial lengths. This size is roughly the full width of the thin current sheet, and is comparable to the inter-spacecraft separation $(\sim 2,000 \mathrm{~km})$. For comparison, the gyroradius of
127-keV (highest energy observed) electrons in a $10 \mathrm{nT}$ magnetic field is $\sim 85 \mathrm{~km}$, much smaller than the estimated size of the magnetic island.

The electron density is highly compressed within islands (black curves in Fig. 3c,f,i, and l). Such density compression, bipolar $B_{z}$ and single-peaked and double-peaked $B_{y}$ are all features of the observed islands (Fig. 3m-p show a multispacecraft view of a three-dimensional island). These features are generic signatures of two-dimensional magnetic islands, as demonstrated by our Hall magnetohydrodynamic simulations of collisionless reconnection (Fig. 4), and $\mathrm{PIC}^{11,17}$ and hybrid simulations ${ }^{18}$. High-time-resolution data of the electron fluxes (16 times higher resolution than those shown in Fig. 3a,d,g,j) for energies greater than $50 \mathrm{keV}$ reveal that energetic electron fluxes (red curves in Fig. 3c,f,i,l) peak at the density compression sites within islands (dotted cyan lines mark seven magnetic islands that have the strongest density compression and corresponding energetic electron fluxes). This key finding establishes a direct link between energetic electrons and magnetic islands, and suggests that the dominant acceleration mechanism and the 

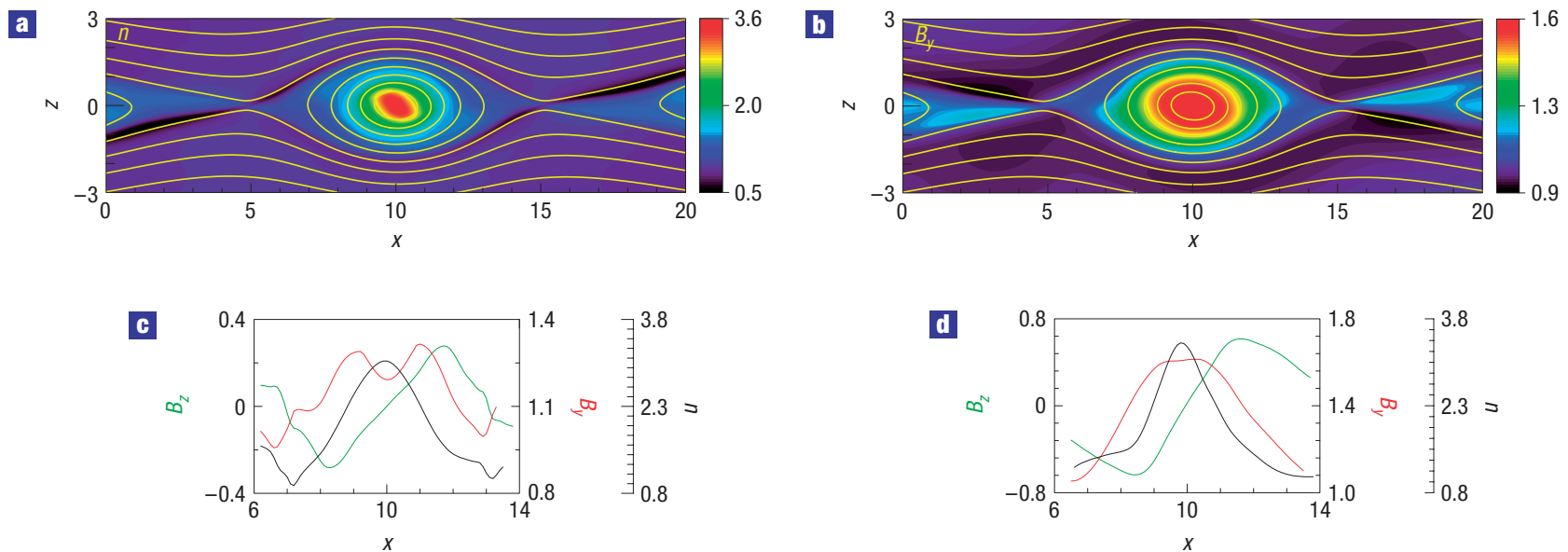

Figure 4 Generic features of a magnetic island as seen in Hall magnetohydrodynamic simulations. a, The density peaking within the island. The magnetic field lines (background yellow curves) show two X lines, with an island in between. $\mathbf{b}$, The out-of-plane magnetic field $B_{y}$ is enhanced within the island. c,d, Snapshots of cuts (along the $z=0.056 d_{i}$ lines in a,b) of $B_{y}$ (red), $B_{z}$ (green) and the density ( $n$, black) across the island showing bipolar $B_{z}$, single- and double-peaked $B_{y}$ and compressed density within the island, qualitatively resembling the island features shown in Fig. $3 n-p$. a,b and $\mathbf{d}$ are snapshots taken at $t=20.66 \tau_{\mathrm{A}}$, and $\mathbf{c}$ a snapshot at $t=17.44 \tau_{\mathrm{A}}$, where $\tau_{\mathrm{A}}$ is the Alfvén transit time across $5 d_{i}$.

physics that causes density compression within islands are intimately related. Note that the electron energization region is more localized than the density compression region, and stronger density compression does not necessarily correspond to higher fluxes of energetic electrons, indicating that the energetic electrons are not produced simply by a compression of the background electrons.

Energetic electrons are also observed in electron-current sheets where the reconnection $\mathrm{X}$ lines reside. Two secondary peaks in the electron flux (Fig. 3d,j) correspond to the two electron-current sheet crossings (marked with dotted brown lines and labelled as 'ecs' in Fig. 3; electron-current sheets are identified on the basis of d.c. electric fields that are normal to the current sheet and of the scale of a few electron inertial lengths ${ }^{19}$ ), consistent with acceleration by the reconnection electric field at X lines. Note that the electrons observed at ecs are much lower in energy, and are one order of magnitude smaller in flux, than those observed within magnetic islands.

Our results indicate that quantitative knowledge on the formation and dynamics of magnetic islands is essential in developing a complete picture of electron acceleration during reconnection. The multispacecraft observations presented above not only provide evidence for the link between energetic electrons and magnetic islands, but also constrain the spatial extent of magnetic islands and their corresponding electron bursts. The structure of the observed islands necessitates a three-dimensional description. In the realm of solar flares, structures that are consistent with multiple magnetic islands in current sheets above flaring arcades have been reported ${ }^{20}$. Data from high-resolution solar X-ray telescopes on board the RHESSI and HINODE spacecraft may shed light on whether there are energetic electrons within these structures during solar flares.

\section{METHODS}

\section{IDENTIFICATION OF MAGNETIC ISLANDS}

One-dimensional cuts (spacecraft trajectories) of the three-dimensional coherent magnetic structures encountered by the Cluster spacecraft show features that are consistent with signatures of magnetic islands established on the basis of two-dimensional models. The observed structures are identified as magnetic islands on the basis of the following criteria: (1) bipolar $B_{z}$ involving a sign change (Fig. 2b,e); (2) electron-density enhancement within bipolar $B_{z}$ (Fig. 2c,f); (3) electron distribution functions within bipolar $B_{z}$ show hot electrons with no appreciable temperature anisotropy in the directions parallel and perpendicular to the magnetic field (data not shown). The second and third criteria distinguish a bipolar $B_{z}$ due to the passage of an island interior from that due to a reconnection $\mathrm{X}$ line. The third criterion is based on the fact that electrons in the inflow near the $\mathrm{X}$-line region are much hotter in the direction parallel to the magnetic field than in the perpendicular direction, whereas in the reconnection region such as the interior of islands the two temperatures are roughly the same ${ }^{19,21,22}$. Time-dependent magnetic fluctuations other than the island perturbations can at times obscure the bipolar $B_{z}$ signature. As a consequence, some of the $B_{z}$ pulses shown in Fig. 2 are not observed by both Cluster 2 and 4 as a qualified bipolar pulse. Moreover, if islands are undergoing coalescence, although criteria 2 and 3 will be met, the nonlinear interaction between islands will prevent the first criterion being satisfied by both islands.

\section{HALL MAGNETOHYDRODYNAMIC SIMULATION}

The simulation that produces the result shown in Fig. 4 is a two-dimensional forced reconnection in a Harris sheet equilibrium with an electron and ion density $n(x, z)=n_{0}+n_{c} \operatorname{sech}^{2}\left(z / L_{c}\right)$, where $n_{0}=0.5 \mathrm{~cm}^{-3}, n_{c}=2 n_{0}$ and $L_{c}=0.5 d_{i}$, where $d_{i}=1.19 \times 10^{3} \mathrm{~km}$ is the ion inertial length. The initial magnetic field is given by $\mathbf{B}=-B_{0} \tanh \left(z / L_{c}\right) \hat{x}+B_{y_{0}} \hat{y}$, where $B_{y 0}=B_{0}=10 \mathrm{nT}$. This initial configuration is driven by inward flows at the upper and lower boundaries. The electrons and ions are assumed to be isothermal, with the temperature $T_{0}=124 \mathrm{eV}$. The system is open along $x$. The initial current sheet thins down considerably, and together with the initial inflow profile results in two reconnection $\mathrm{X}$ lines that contain a magnetic island in between them. The island has a compressed plasma density and an enhanced core magnetic field as shown in Fig. 4. Detailed descriptions of the code are given in ref. 23 and references therein. Although the results presented in Fig. 4 were obtained with a sizable initial guide field, we note that the initial guide field $B_{y_{0}}$ can be reduced to $3 \%$ of $B_{0}$, whereby the resulting density still peaks at the island centre, and the core field $B_{y}$ has a well-defined centre peak within the island. The development of the $B_{y}$ centre peak takes a longer time when the initial guide field is smaller.

\section{MULTI-SPACECRAFT ANALYSIS OF ISLAND PROPERTIES}

The cross-correlation between the $B_{y}$ observed by Cluster 2 and 4 at around the time 09:47:40 u t yields a time delay of $\sim 3 \mathrm{~s}$. We assume that the island is localized in $x$ and $z$, extended in $y$, and the extension axis is along $y$. Such an 
assumed structure is what we would expect for filaments owing to the breakup of a current sheet on the $x-y$ plane with its currents flowing along $y$. The propagation velocity of the island is estimated to be $\sim 500 \mathrm{~km} \mathrm{~s}^{-1}$ away from the Earth, on the basis of the $\sim 1,500 \mathrm{~km}$ separation in $x$ between Cluster 2 and 4 . Using the time width of $\sim 5 \mathrm{~s}$ for the Cluster $4 B_{y}$ pulse (Fig. 3p), the size of the island in the $x$ direction is estimated to be $\sim 2,500 \mathrm{~km}$. The positive $z$ extent (above the current sheet) of the island is larger than $1,000 \mathrm{~km}$ (because Cluster 2-4 all crossed this same island, and the $z$ range of these three spacecraft positions is $1,000 \mathrm{~km}$ ), and smaller than $2,000 \mathrm{~km}$ (because Cluster 1 did not encounter this island). Assuming the island centre is symmetric with respect to the current sheet, the $z$ width and the $x$ width of the island are similar, implying that the island is quite circular in the reconnection plane. Both the island speed and size are of the same order as those obtained previously in a case study (speed $\sim 700 \mathrm{~km} \mathrm{~s}^{-1}$; size $\sim 6,400 \mathrm{~km}$ ), where all four Cluster spacecraft observed highly correlated magnetic structures ${ }^{24}$.

\section{Received 6 March 2007; accepted 1 0ctober 2007; published 11 November 2007.}

\section{References}

1. Bhattacharjee, A. Impulsive magnetic reconnection in the Earth's magnetotail and the solar corona. Annu. Rev. Astron. Astrophys. 42, 365-384 (2004).

2. Masuda, S., Kosugi, T., Hara, H., Tsuneta, S. \& Ogawara, Y. A loop-top hard X-ray source in a compact solar flare as evidence for magnetic reconnection. Nature 371, 495-497 (1994).

3. Tsuneta, S. Structure and dynamics of magnetic reconnection in a solar flare. Astrophys. J. 456, 840-849 (1996)

4. Hurley, K. et al. An exceptionally bright flare from SGR 180620 and the origins of short-duration big gamma-ray bursts. Nature 434, 1098-1103 (2005).

5. Hones, E. W. Jr. Plasma flow in the plasma sheet and its relation to substorms. Radio Sci. 8 979-990 (1973).

6. Lin, R. P. \& Hudson, H. S. Nonthermal processes in large solar flares. Sol. Phys. 50, 153-178 (1976).

7. Lin, R. P., Krucker, S., Hurford, G. J., Smith, D. M. \& Hudson, H. S. RHESSI observations of particle acceleration and energy release in an intense solar gamma-ray line flare. Astrophys. J. 595, L69-L76 (2003)

8. Holman, G. D., Linhui, S., Schwartz, R. A. \& Emslie, A. G. Electron Bremsstrahlung hard X-ray spectra, electron distributions, and energetics in the 2002 July 23 solar flare. Astrophys. J. 595 L97-L101 (2003).

9. Drake, J. F., Swisdak, M., Che, H. \& Shay, M. A. Electron acceleration from contracting magnetic islands during reconnection. Nature 443, 553-556 (2006).

10. Oieroset, M., Lin, R. P., Phan, T. D., Larson, D. E. \& Bale, S. D. Evidence for electron acceleration up to $\sim 300 \mathrm{keV}$ in the magnetic reconnection diffusion region of Earth's magnetotail. Phys. Rev. Lett. 89, 195001 (2002).

11. Drake, J. F., Shay, M. A. \& Thongthai, W. Production of energetic electrons during magnetic reconnection. Phys. Rev. Lett. 94, 095001 (2005).
12. Hoshino, M., Mukai, T., Terasawa, T. \& Shinohara, I. Suprathermal electron acceleration in magnetic reconnection. J. Geophys. Res. 106, 25979-25997 (2001).

13. Pritchett, P. L. Relativistic electron production during driven magnetic reconnection. Geophys. Res. Lett. 33, L13104 (2006).

14. Escoubet, C. P., Schmidt, R. \& Goldstein, M. L. Cluster-science and mission overview. Space Sci. Rev. 79, 11-32 (1997)

15. Kistler, L. M. et al. Contribution of nonadiabatic ions to the cross-tail current in an $\mathrm{O}^{+}$dominated thin current sheet. J. Geophys. Res. 110, A06213 (2005).

16. Ma, Z. W. \& Bhattacharjee, A. Sudden disruption of a thin current sheet in collisionless Hall magnetohydrodynamics due to secondary tearing and coalescence instabilities. Geophys. Res. Lett. 26, 3337-3340 (1999).

17. Drake, J. F., Swisdak, M., Schoeffler, K. M., Rogers, B. N. \& Kobayashi, S. Formation of secondary islands during magnetic reconnection. Geophys. Res. Lett. 33, L13105 (2006).

18. Karimabadi, H., Krauss-Verban, D., Omidi, N. \& Vu, H. X. Magnetic structure of the reconnection layer and core field generation in plasmoids. J. Geophys. Res. 104, 12313-12326 (1999).

19. Chen, L.-J. et al. DC electric fields and electron distribution functions within the diffusion region of magnetotail reconnection: Comparison between Cluster observations and PIC simulations. American Geophysical Union (2006), Fall Meeting.

20. McKenzie, D. E. \& Hudson, H. S. X-ray observations of motions and structure above a solar flare arcade. Astrophys. J. 519, L93-L96 (1999).

21. Cattell, C. et al. Cluster observations of electron holes in association with magnetotail reconnection and comparison to simulations. J. Geophys. Res. 110, A01211 (2005).

22. Schmitz, H. \& Grauer, R. Kinetic Vlasov simulations of collisionless magnetic reconnection. Phys. Plasmas 13, 092309 (2006)

23. Yang, H., Jin, S. P. \& Zhou, G. C. Density depletion and Hall effect in magnetic reconnection. J. Geophys. Res. 111, A11223 (2006).

24. Slavin, J. A. et al. Cluster electric current density measurements within a magnetic flux rope in the plasma sheet. Geophys. Res. Lett. 30, 1362-1365 (2003).

25. Pedersen, A. et al. Four-point high time resolution information on electron densities by the electric field measurements (EFW) on Cluster. Ann. Geophys. 19, 1483-1489 (2001).

26. Imada, S. et al. Energetic electron acceleration in the downstream reconnection outflow region. J. Geophys. Res. 112, A03202 (2007).

\section{Acknowledgements}

We thank K. Donahue for her assistance in figure preparation. Research at the UNH was supported by NASA SECGIP04-0025-0171, NSF ATM-0425806 and DoE DE-F902-05ER59832. Research at MPI, Lindau, was supported by DLR grant 50 OC 0003 , and that in the UK by PPARC.

Correspondence and requests for materials should be addressed to L.-J.C.

\section{Author contributions}

L.-J.C. identified the correlation between energetic electrons and magnetic islands, carried out data analysis and comparison with simulation results and wrote the paper. A.B. oversaw the research project, and facilitated data-simulation comparison. P.A.P.-Q., S.I., S.M., P.W.D., B.L., Y.K., A.V., A.F and E.G. provided Cluster data and data processing. H.Y. carried out Hall magnetohydrodynamic simulations and N.B. PIC simulations to elucidate kinetic properties of magnetic islands during reconnection. A.B., P.A.P.-Q., H.Y., N.B., S.I. and B.L. discussed the results with L.-J.C., and commented on the paper.

Reprints and permission information is available online at http://npg.nature.com/reprintsandpermissions/ 